\title{
Sympathetic activation by the cold pressor test does not increase the muscle force generation capacity
}

Silvestro Roatta and Dario Farina

J Appl Physiol 110:1526-1533, 2011. First published 31 March 2011;

doi: 10.1152/japplphysiol.00039.2011

You might find this additional info useful...

This article cites 38 articles, 17 of which you can access for free at:

http://jap.physiology.org/content/110/6/1526.full\#ref-list-1

Updated information and services including high resolution figures, can be found at: http://jap.physiology.org/content/110/6/1526.full

Additional material and information about Journal of Applied Physiology can be found at: http://www.the-aps.org/publications/jappl

This information is current as of November 12, 2012. 


\title{
Sympathetic activation by the cold pressor test does not increase the muscle
}

\section{force generation capacity}

\author{
Silvestro Roatta ${ }^{1}$ and Dario Farina ${ }^{2}$ \\ ${ }^{1}$ Dipartimento di Neuroscienze, Sezione di Fisiologia, Università di Torino, Torino, Italy; and ${ }^{2}$ Department of \\ Neurorehabilitation Engineering, Bernstein Center for Computational Neuroscience, University Medical Center Göttingen, \\ Georg-August University, Göttingen, Germany
}

Submitted 11 January 2011; accepted in final form 28 March 2011

Roatta S, Farina D. Sympathetic activation by the cold pressor test does not increase the muscle force generation capacity. $J$ Appl Physiol 110: 1526-1533, 2011. First published March 31, 2011; doi:10.1152/japplphysiol.00039.2011.-A positive inotropic action by the sympathetic nervous system on skeletal muscles has been observed and investigated in animal and in vitro studies. This action provided a theoretical basis for the putative ergogenic action of catecholamines and adrenergic agonists, although there is no clear evidence of this effect in humans. The aim of this study was to investigate the occurrence of inotropic effects associated to physiological sympathetic activation in healthy subjects. The muscle force capacity was investigated in the tibialis anterior ( $n=9$ subjects) and in the soleus $(n=9)$ muscles electrically stimulated with single pulses and double pulses with variable interspike interval (4-1,000 ms) and short pulse trains (frequency: 5-14 Hz) before, during, and after sympathetic activation by the cold pressor test (CPT). CPT significantly decreased by $10.4 \pm 7.2$ and $10.6 \pm 4.4 \%$ the force produced by single and double pulse stimulation, respectively, and produced smaller decreases in the force obtained by train stimulation in the tibialis anterior, while no significant changes were observed in either type of contraction in the soleus muscle. CPT failed to induce any increase in the force capacity of the investigated muscles. The prevalent decrease in force evidenced in this study supports the concept that the weakening sympathetic action on type I fiber, already shown to occur in humans, prevails over the putative potentiating action.

inotropic effect; electrical stimulation; twitch force; catecholamines; adrenaline

SYMPATHETIC ACTIVITY IS KNOWN to support motor function by acting at different levels, including the cardiovascular, respiratory, and motor systems. The release of catecholamines in the blood accompanies physical exercise depending on its extent and duration (39), and catecholamine outflow was found to be correlated with motor performance (11). In addition, administration of sympathomimetics, particularly $\beta_{2}$-adrenergic agonists, such as salbutamol, have been shown to improve motor performance in different types of tasks $(8,20,32,34)$. To explain this ergogenic effect, a specific potentiating action on skeletal muscle contractility is often invoked (36). Indeed, a positive inotropic effect of epinephrine (Epi) and adrenergic agonists on skeletal muscles has been well documented in anesthetized animals as well as in isolated muscles and fiber bundles. This effect has been found to be mediated by $\beta_{2^{-}}$ adrenergic receptors leading to increased $\mathrm{Ca}$ release from the sarcoplasmatic reticulum $(1,3,5,12,35)$. There are, however, studies in which administration of Epi or $\beta_{2}$-agonists failed to

Address for reprint requests and other correspondence: S. Roatta, Dept. Neuroscience, Physiology Div., Università di Torino, c.so Raffaello 30, 10125 Torino, Italy (e-mail: silvestro.roatta@unito.it). induce contractility potentiation (21) or improvement in motor performance $(7,19)$.

One possible factor behind these conflicting results may be the complexity of the adrenergic action, differentially affecting the contractile machinery of type I and type II muscle fibers. A positive inotropic effect is indeed mainly exhibited by type II fibers, whereas the prevailing effect in type I fibers is a positive lusitropic effect, i.e., a shortening of the duration of the twitch force due to increased relaxation rate $(3,29)$. Notably, while the former effect mediates a potentiation of the contraction, the latter corresponds to a weakening action because shorter lasting twitches result in diminished twitch summation and therefore in a lower average force level in sustained subtetanic contractions $(3,28,29)$. In addition, the weakening effect (shortening of the twitch force) in type I fibers could be experimentally elicited at "low" concentrations of the adrenergic agonist, while the potentiating effect on type II fibers required concentrations 4-12 times greater (1, 3), casting doubts on its physiological relevance (3).

Although several animal experiments and in vitro studies have investigated the modulation of muscle contractility by sympathomimetics, little evidence exists on the adrenergic modulation of muscle contractility in humans in physiological conditions. Only very recently, evidence of a weakening effect on type I fibers, as reported in animal studies, was provided for low-threshold (presumably composed of type I fibers) motor units during physiological sympathetic activation by the cold pressor test (CPT) in healthy subjects (28). Conversely, whether endogenous catecholamines effectively mediate an inotropic effect in skeletal muscles (specifically type II fibers) in humans under physiological conditions remains to be demonstrated and the possible implication in motor control and performance remains a matter of debate.

The aim of this study was to investigate the occurrence of a positive inotropic effect in skeletal muscles of healthy subjects during physiological sympathetic activation by the CPT. In our previous study (28), the weakening effect on type I fibers was investigated during low-level voluntary contractions in which only few low-threshold, presumably type I, motor units are recruited. This condition allows the twitch force of single motor units to be estimated by means of the spike-triggering averaging technique. This approach is, however, technically inapplicable to high-threshold type II motor units. The current study was thus based on electrically elicited contractions. The putative sympathetic induced increase in muscle force capacity should be related to the fraction of type II fibers in the muscle. To test this hypothesis, the study was conducted on two muscles with a different fiber type composition, the tibialis anterior muscle ( $70 \%$ type I, $30 \%$ type II) and the soleus 
muscle (85\% type I, $15 \%$ type II) (16). Due to its larger fraction of type II fibers, a larger positive inotropic effect was expected in the tibialis anterior muscle.

\section{MATERIALS AND METHODS}

The study consisted of two experiments in which electrically elicited isometric contractions were measured in the tibialis anterior (experiment 1) and in the soleus (experiment 2) muscles before, during, and after activation of the sympathetic nervous system by the CPT. Unless otherwise specified, the descriptions of the methods refer to both experimental conditions.

Subjects. Eleven (age: $27.3 \pm 4.1 \mathrm{yr}$; height: $173 \pm 9 \mathrm{~cm}$; and weight: $67 \pm 9 \mathrm{~kg}$ ) and 12 (age: $28.3 \pm 3.8 \mathrm{yr}$; height: $174 \pm 9 \mathrm{~cm}$; and weight $70 \pm 12 \mathrm{~kg}$ ) healthy men participated in experiment 1 and 2 , respectively. They were recruited among the student population and the research staff at the University Campus, none of them practicing sport at agonistic level. The experimental protocols, approved by the local ethical committee (N-20070017), were in accordance with the Declaration of Helsinki. All subjects gave their informed consent before participation in the experiments.

Experimental setup. Subjects were asked to refrain from meals and coffee in the hour before the beginning of the experiment. The subject was seated on a dental chair of adjustable height with his right foot fixed to a foot plate. The positions of chair and foot plate were adjusted so that the knee and ankle joint angles, respectively, were $\sim 100^{\circ}$ and $70^{\circ}$ (experiment 2 ) and both $90^{\circ}$ (experiment 1 ). The leg was stabilized by Velcro straps and by a vacuum-packed kapok-filled pillow (Ambu, Kristianstad, Sweden) that prevented side movements of the leg. Care was taken in tightly fixing the foot to the foot plate. For this purpose, no padding was used to avoid damping of the torque measurement at the ankle joint.

Torque and electromyogram. The footplate was equipped with a strain gauge providing a signal proportional to the elastic deformation. This signal was amplified (Amplifier Unit LAU 73.1; Soemer, Lennestadt, Germany) and used to measure the absolute torque level produced at the ankle $(1.05 \mathrm{Nm} / \mathrm{V}$; bandwidth $0-50 \mathrm{~Hz})$.

Surface electromyogram (EMG) signals were recorded using bipolar circular electrodes (1-cm diameter, $2 \mathrm{~cm}$ apart) placed along the direction of the muscle fibers on the tibialis anterior muscle, $\sim 2 \mathrm{~cm}$ lateral to the tibial bone and $5 \mathrm{~cm}$ distal to the tibia tuberosity (experiment 1), and on the soleus muscle below the gastocnemius muscle, $2 \mathrm{~cm}$ lateral to the tendon (experiment 2). The ground electrode was placed at the ankle.

Blood pressure and subjective pain ratings. Systolic and diastolic blood pressures were measured with a digital blood pressure meter (UA-751; Simonsen \& Weel). The manometer cuff was released after each measure, and the arm was raised up a few seconds for quick recovery of perfusion regimen in the arm.

The pain intensity was continuously scored by the subjects on an electronic $10-\mathrm{cm}$ visual analog scale (VAS) with the lower extreme labeled "no pain" and the upper extreme labeled "most pain imaginable."

EMG, torque, and VAS were concurrently sampled (12-bit A/D conversion, $2 \mathrm{kHz}$ sampling frequency) and stored on a PC.

Electrical stimulation. Electrical stimulation was provided by a voltage-controlled current source stimulator (NoxiSTIM; JNI Biomedical; Aalborg, Denmark). In experiment 1, the stimulation of the tibialis anterior muscle was obtained by stimulating the peroneal nerve, the cathode (electrode diameter: $2 \mathrm{~cm}$ ) being placed just above the fibula neck and the anode $(3 \times 3 \mathrm{~cm})$ at the patella. In experiment 2 , the calf muscle was stimulated by a cathode electrode (diameter of $2 \mathrm{~cm}$ ) placed on the tibial nerve at the popliteal fossa. To reduce the contribution of the gastrocnemius muscle, the knee was flexed at $\sim 100^{\circ}$. However, in this position the electrode nerve coupling is impaired, compared with the knee-extended position. Therefore, a custom device was fixed to the thigh and exerted an adjustable pressure on the cathode electrode at the popliteal fossa to improve the effectiveness and reliability of the nerve stimulation. The anode electrode $(3 \times 3 \mathrm{~cm})$ was placed at the patella. The anode position was adjusted to avoid unwanted contractions of antagonist muscles during the stimulation, which was detected by monitoring EMG activity on the tibialis anterior muscle.

For each subject, the stimulation intensity evoking the maximum compound muscle action potential was determined. However, in some cases, a supramaximal intensity of stimulation was reported to be painful. Because it was important to avoid preventive pain-induced sympathetic activation related to the stimulation, in those cases we adopted the maximum stimulation intensity that was nonpainful.

Procedures. The types of stimulation performed were single pulses, doublets, and pulse trains. The stimulation pattern for single stimuli and doublets consisted of a sequence of 22 alternated single and paired pulses separated by 1-s interval. The paired pulses (doublets) had an interspike interval (ISI) ranging between 4 and $1,000 \mathrm{~ms}(4,8$, $12,15,20,30,50,75,100,125,150,175,200,225,250,300,400$, 500,750 , and $1,000 \mathrm{~ms}$ ) according to a protocol adopted in previous studies $(17,18)$. This sequence of pulses was followed by four pulse trains of $5 \mathrm{~s}$ in duration, separated by 5 -s intervals. The pulse trains had frequencies 5, 8, 10, and $12 \mathrm{~Hz}$ (Fig. 1B).

The set of stimulations was repeated seven times, corresponding to three control conditions ( $\mathrm{C} 1, \mathrm{C} 2$, and $\mathrm{C} 3$ ), one condition of sympathetic activation $(\mathrm{CPT})$, and three recovery conditions (P1, P2, and P3) (Fig. 1A). Each recording condition was separated by 5 -min intervals.

In the CPT condition, the left hand was immersed in iced water $\left(3-4^{\circ} \mathrm{C}\right)$, stirred by a peristaltic pump, for $4 \mathrm{~min}$. The subjects could withdraw the hand from the water if the pain became unbearable, in which case the data were excluded from the analysis. In study 2 only, in one of the control conditions the left hand was immersed in water at $32-35^{\circ} \mathrm{C}$ (neutral condition) and the sequence of the control and neutral conditions was randomized.

Systolic and diastolic blood pressures were measured during the control condition just after $\mathrm{C} 2$, immediately after the CPT, and during the recovery just after P2 (see black dots in Fig. 1A).
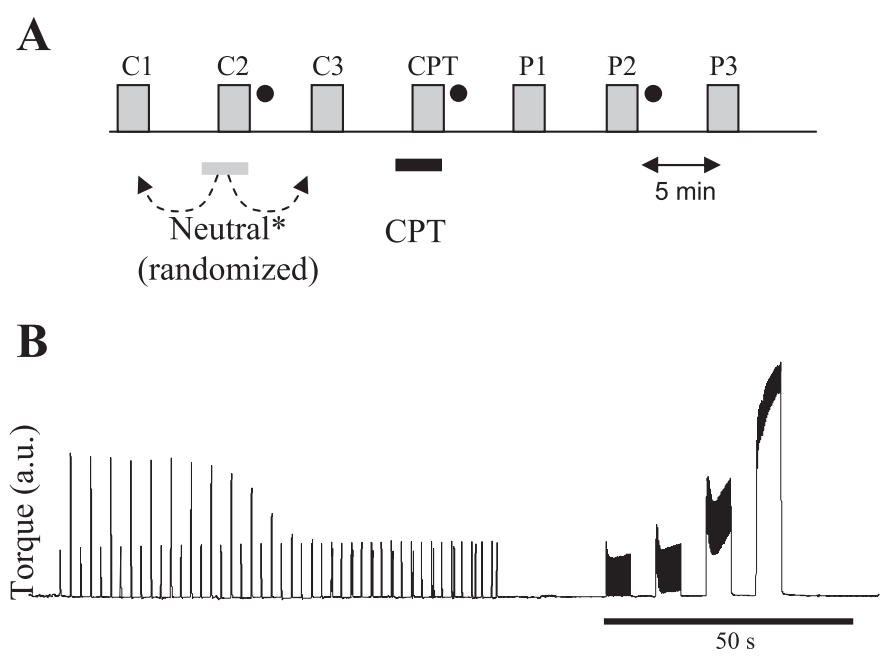

Fig. 1. Experimental protocol. A: same protocol was applied for the stimulation of the peroneal nerve (experiment 1 ) and the posterior tibialis nerve (experiment 2). The same stimulation pattern was repeated 7 consecutive times: before (C1, C2, and C3), during [cold pressor test (CPT)], and after (P1, P2, and P3) administration of the (CPT, left hand immersed in icy water for $4 \mathrm{~min}$ ). *In a randomized control condition $(\mathrm{C} 1, \mathrm{C} 2$, or $\mathrm{C} 3)$, the left hand was also immersed in warm water (neutral; experiment 2, only). Black dots indicate measurement of arterial blood pressure. $B$ : torque developed by the stimulation pattern in a control stimulation in 1 subject. Stimulation pattern consists of a sequence of 22 single pulses interleaved with 22 double pulses (doublets) with interspike interval (ISI) increasing from 4 to $1,000 \mathrm{~ms}$, followed by 4 bursts at constant frequency of $5,8,10$, and $12 \mathrm{~Hz}$, lasting $5 \mathrm{~s}$. 
Signal analysis. Values of systolic and diastolic blood pressure and VAS ratings were averaged over each condition.

Time-to-peak (TTP), half-relaxation time (HRT), and peak amplitude (PA) were computed from the average of 21 single twitch torques, following the first stimulus. The twitch torque elicited by the first stimulus was excluded because it was systematically smaller than all the others. The same parameters were extracted from the doublet stimulation for the second stimulus in each pair of stimuli. The PA value was identified as the maximum torque increase (with respect to the prestimulation level) reached after the stimulating pulse. Since no changes in nerve conduction velocity were expected, TTP was more conveniently computed as the interval between the stimulation pulse and the time instant corresponding to the torque peak, rather than between the onset of torque development and the torque peak. HRT was computed as the interval between the torque peak and the instant in which the torque was reduced to half its peak value.

From the pulse trains, the average torque and the amplitude of torque oscillations were extracted. The average torque was computed by averaging the torque signal over the last $1 \mathrm{~s}$ of stimulation during the pulse train. The amplitude of torque oscillation was obtained as the peak-to-peak amplitude of the torque signal, as average value over the last $1 \mathrm{~s}$ of stimulation. These values were normalized with respect to the average of all conditions before being averaged over subjects.

Statistical analysis. For both experiments, nonparametric statistical analysis was adopted because the normality tests failed for some of the analyzed variables (diastolic blood pressure in control condition, experiment 1 and 2). The Kruskal-Wallis ANOVA and Mann-Whitney $U$-test were used to compare blood pressure changes and VAS score in the two studies. One way ANOVA for repeated measures was used to assess an effect of condition $(\mathrm{C} 1, \mathrm{C} 2, \mathrm{C} 3, \mathrm{CPT}, \mathrm{P} 1, \mathrm{P} 2$, and $\mathrm{P} 3)$ on the measured variables. When ANOVA was significant $(P<$ $0.05)$, pair-wise comparisons were tested by the Newman-Keuls post hoc test. Values are presented as means \pm SD in the text and as means $\pm \mathrm{SE}$ in the figures.

\section{RESULTS}

Experiment 1: stimulation of peroneal nerve. Two out of the eleven recruited subjects had to be discarded because of unstable force recording due to involuntary contractions during the CPT. The results are collected from the remaining nine subjects.

On average, the intensity of stimulation of the peroneal nerve was $113.0 \pm 9.3 \%$ of the intensity producing the maximum amplitude in the EMG response to single stimuli (M wave) in the tibialis anterior muscle (range: 95-120\%).

CPT evoked a persistent painful sensation that outlasted the duration of the test. The peak VAS score was $4.5 \pm 1.9$ (range: 2.7-8.7). The painful sensation vanished in all subjects before P2.

CPT produced an increase in diastolic blood pressure from $73.8 \pm 6.0$ to $89.0 \pm 9.2 \mathrm{mmHg}(P<0.01)$ and systolic blood pressure from $109.0 \pm 9.7$ to $127.6 \pm 11.0 \mathrm{mmHg}(P<0.01)$. Both variables returned to control values when reassessed after P2 condition (diastolic: $75.7 \pm 5.6 \mathrm{mmHg}$; systolic: $113.2 \pm$ $8.2 \mathrm{mmHg})$.

The effect of CPT on the torque twitch evoked by single electrical stimuli is exemplified by the recordings from a representative subject (Fig. 1A). Group effects across all subjects on AMP, TTP, and HRT are shown in the bar diagrams in Fig. 2, $B-D$. We observed a slight increase in AMP during the three control conditions: $\mathrm{C} 3$ being higher then $\mathrm{C} 1$ by $8.2 \pm$ 9.1\% $(P<0.01)$, possibly due to postcontraction potentiation mechanisms. However, AMP was significantly reduced by $10.4 \pm 7.2 \%(P<0.01)$ during $\mathrm{CPT}$ with respect to $\mathrm{C} 3$. A gradual recovery of twitch amplitude was observed in the recovery conditions, with $\mathrm{P} 3$ being significantly different from $\mathrm{CPT}$ and matching the value of C3. Conversely, no significant changes were instead observed in the time course of TTP $(116 \pm 13 \mathrm{~ms}$ in $\mathrm{C} 1$; Fig. $2 C)$ and HRT $(73 \pm 17 \mathrm{~ms}$ in $\mathrm{C} 1$; Fig. 2D).

The analysis of the twitch torque produced by the second of two spikes administered with variable ISI is shown in Fig. 3. For ISI $<20 \mathrm{~ms}$, the two twitches are fused together and the amplitude is almost independent of the ISI. With increasing ISI $>20 \mathrm{~ms}$, the two twitches begin to split and the amplitude of
Fig. 2. Stimulation of the peroneal nerve with single pulses (experiment 1). A: tracing of the twitch torque from a representative subject in 3 conditions ( $\mathrm{C} 1, \mathrm{CPT}$, and $\mathrm{P} 3)$. Each trace is the average of 20 single twitches. $B, C$, $D$ : effect of CPT on twitch amplitude (AMP, $B$ ), time to peak (TTP; $C$ ), and half relaxation time (HRT; $D)$. AMP values were normalized with respect to the average over all conditions. $* P<0.01$, significantly different from $\mathrm{C} 3, \mathrm{P} 2$, and $\mathrm{P} 3(n=9)$.
A

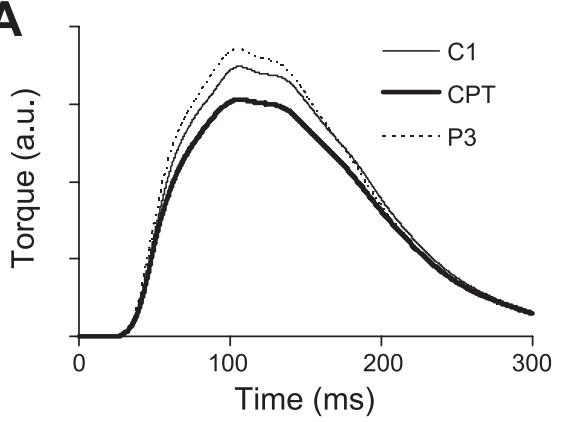

C

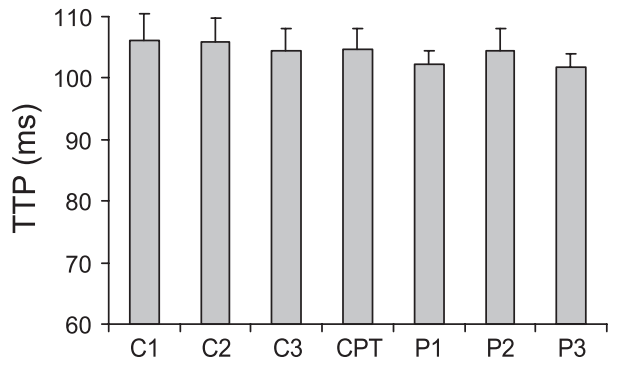

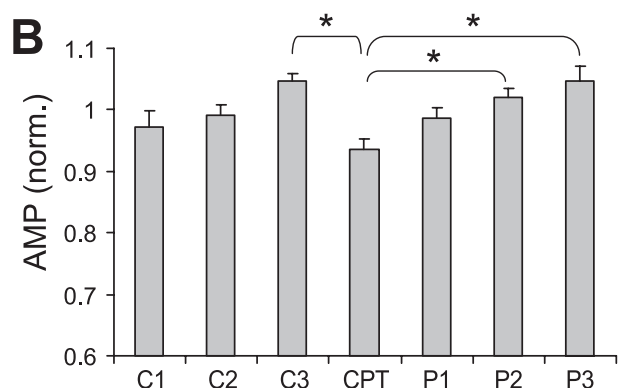

D

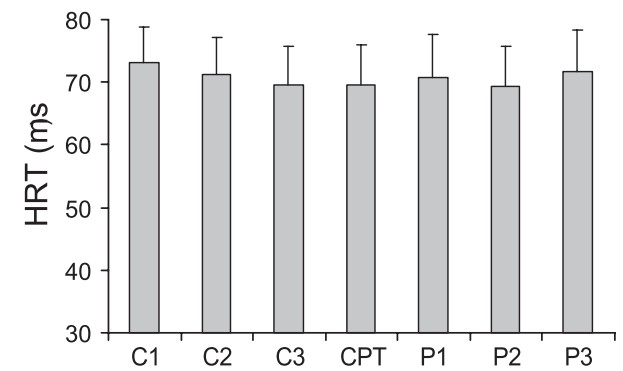



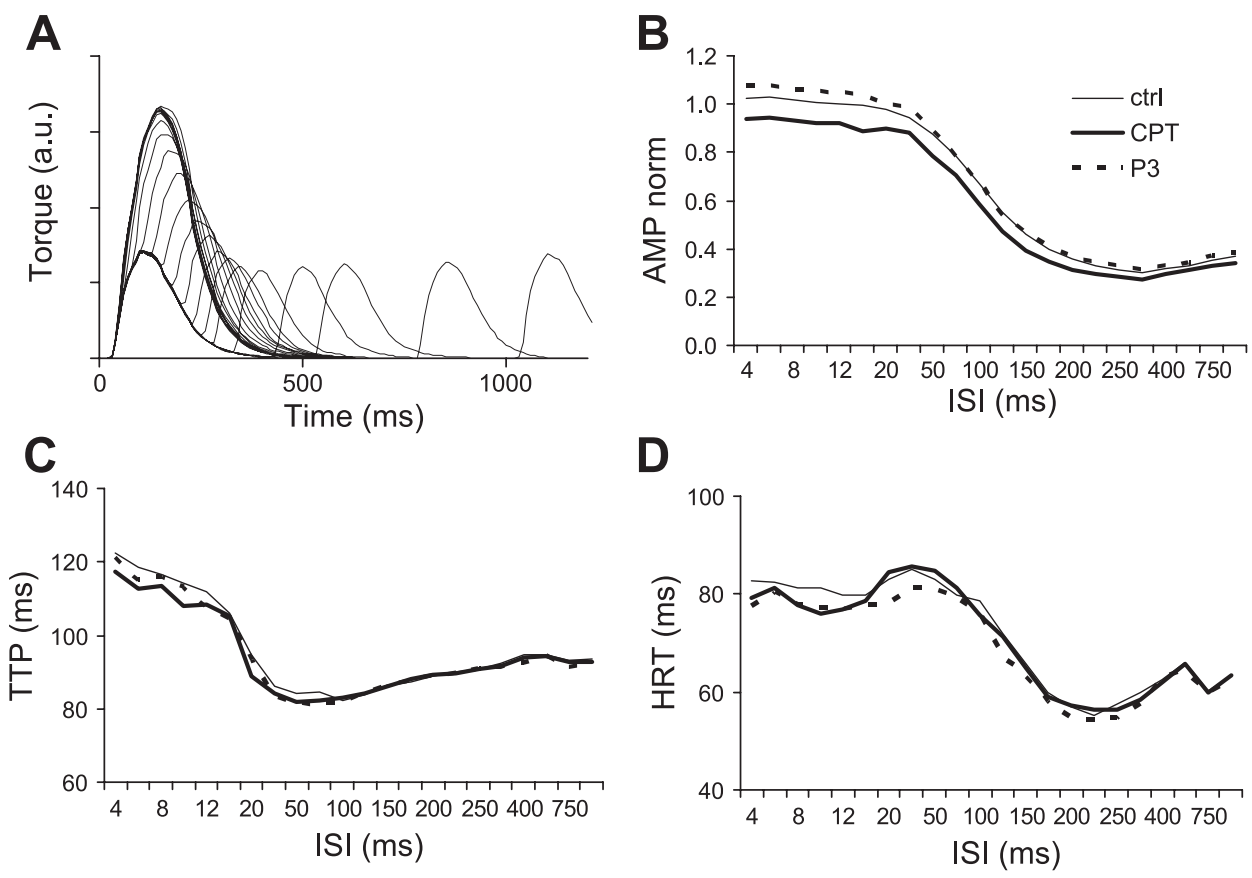

Fig. 3. Analysis of torque developed by stimulation with double pulses of the peroneal nerve (experiment 1). A: superimposition of the torque produced by the 21 doublets at increasing ISI in 1 subject in a control condition. $B, C$, and $D$ : effect on amplitude, time to peak, and half relaxation time of the torque produced by the second pulse in the doublet is displayed vs. the ISI (ms) for 3 conditions. Each trace is the average of all individual traces $(n=9)$, and ctrl is the average of the 3 control conditions. Abbreviations are as in Fig. 2.

the second one starts to fall (Fig. 3A). Above 300-400 ms, the second twitch is completely separated from the first one and its characteristics tend to approach the characteristics of the single twitch described in Fig. 2. With ISI $>30 \mathrm{~ms}$, the two twitches are only partly fused and the peak amplitude (detected after the second pulse) starts to decrease. The TTP and HRT also exhibit a clear dependency on ISI.

The CPT influenced the amplitude of the peak torque produced by the second pulse at all ISIs tested (thick line in Fig. $3 B$ ). The differences were significant for the average peak amplitude of the first three doublets (ISI $=4,6$, and $8 \mathrm{~ms}$ ), which was reduced by $10.6 \pm 4.4 \%$ during the CPT with respect to $\mathrm{C} 3$ and was significantly different from all other conditions $(P<0.01$, except vs. C1: $P<0.05)$. TTP and HRT were not influenced by CPT (Fig. 3, $C$ and $D$ ).

The contractions evoked by burst stimulations were analyzed in terms of the torque reached at the end of the burst and of the amplitude of torque oscillations. On average, the torque developed during CPT was lower than that developed in all other conditions for each of the stimulation frequencies employed; however, the significance level was not reached. The amplitude of torque oscillation during burst stimulation at $5 \mathrm{~Hz}$ exhibited a similar time course as the torque twitch amplitude, i.e., a slight increasing trend between $\mathrm{C} 1$ and C3 (10 $\pm 12 \%)$ and between P1 and P3 $(9 \pm 13 \%)$ but with a decrease between C3 and CPT $(6 \pm 13 \%)$. Similar, but less marked, changes were observed at 8,10 , and $12 \mathrm{~Hz}$, although none of these changes reached statistical significance.

Experiment 2: stimulation of the tibial nerve. One subject had to be excluded because of instability of the force recording, and two other subjects were discarded because of the presence of an $\mathrm{H}$ reflex in response to the electrical stimulation. The results are described for the nine remaining subjects.

Electrical stimulation of the tibial nerve appeared to be relatively more painful than stimulation of the peroneal nerve, and, to avoid pain sensations associated to the electrical stim- ulation, the intensity often had to be reduced below the one producing the maximum $\mathrm{M}$ wave $(89 \pm 10.3 \%$, range: $76-$ $106 \%$ ).

Hand immersion in water at neutral temperature did not evoke a pain sensation (VAS $=0$ in all subjects) while CPT evoked similar effects to those described for experiment 1 . The VAS score peaked at $5.6 \pm 3.1$ (range: $2.3-9.2$ ) during the test and returned to 0 in all subjects at P2. Diastolic blood pressure rose from $73.8 \pm 6.0$ to $89.0 \pm 9.2 \mathrm{mmHg}(P<0.01)$ and systolic blood pressure from $109.0 \pm 9.7$ to $127.6 \pm 11.0$ $\mathrm{mmHg}(P<0.01)$. Both variables returned to control values when reassessed, after P2 condition (diastolic: $75.7 \pm 5.6$ mmHg; systolic: $113.2 \pm 8.2 \mathrm{mmHg}$ ). VAS and blood pressure changes were not significantly different in experiment 2 compared with experiment 1 .

A representative example of the single twitch of calf muscles evoked by stimulation of the tibial nerve is reported in Fig. $4 \mathrm{~A}$. The time course of the twitch torque in the control condition (average of $\mathrm{C} 1-\mathrm{C} 3$ ) was slower than in experiment 1, as observed both for TTP $(124 \pm 19$ vs. $105 \pm 11 \mathrm{~ms} ; P<0.01)$ and for HRT $(99 \pm 23$ vs. $71 \pm 18 \mathrm{~ms} ; P<0.05)$. In one of the control conditions, the left hand was immersed in water at neutral temperature, and on average, the twitch parameters did not depend on the control condition. CPT did not influence the twitch amplitude (Fig. 5B) nor TTP (Fig. 5C) nor HRT (Fig. $5 C)$, although the latter was reduced in seven of the nine subjects during CPT (4\% reduction with respect to the average of $\mathrm{C} 1-\mathrm{C} 3$ ).

An example of the evoked contractions during the stimulation with double pulses is shown in Fig. 5A, while Fig. 5, $B$ and $C$, reports the mean curves for the different conditions. Also for this stimulation paradigm, the parameters did not depend on the condition. As observed for the single twitch, HRT was slightly but not significantly reduced at ISI $<50 \mathrm{~ms}$ (Fig. 5C). Absence of systematic changes in the time course of the contraction is also confirmed by the absence of changes in the 
Fig. 4. Stimulation of the tibial nerve with single pulses (experiment 2). A: tracing of the twitch torque from a representative subject in 3 conditions ( $\mathrm{C} 1, \mathrm{CPT}$, and $\mathrm{P} 3)$. Each trace is the average of 20 single twitches. Effect of CPT on twitch amplitude $(B)$, time to peak $(C)$, and half relaxation time $(D)$. Amplitude values were normalized with respect to the average over all conditions. Abbreviations as in Fig. $2(n=9)$.
A

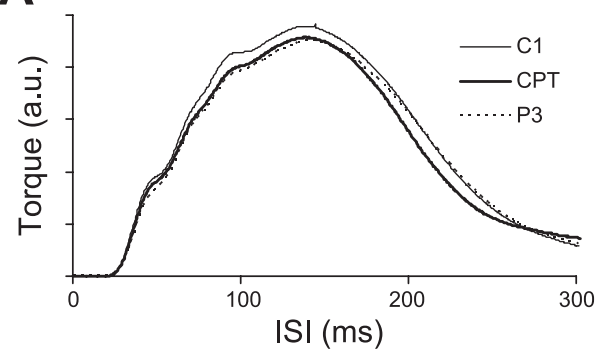

C

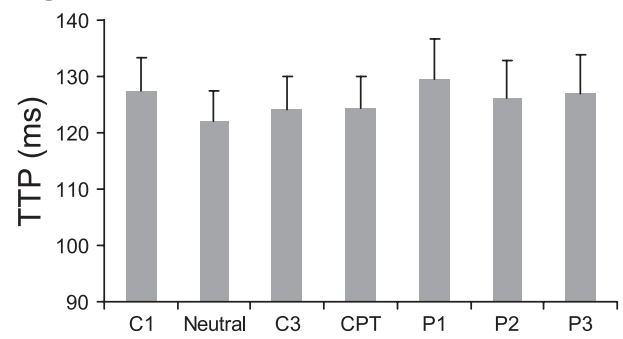

B

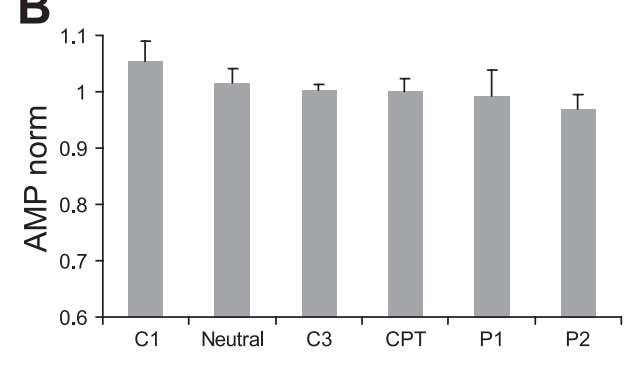

D

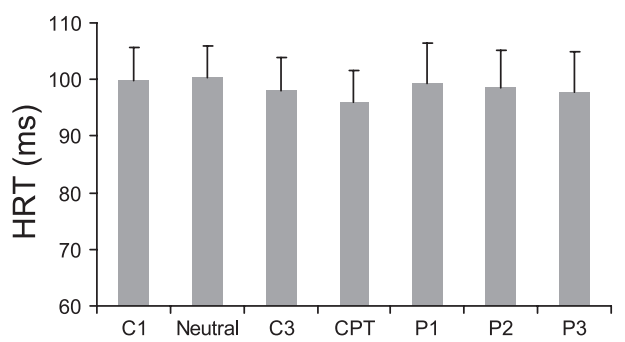

pattern of summation of the double twitches, as indicated by the curves in Fig. $5 B$.

The torque developed by burst stimulation was also unaffected by CPT both in terms of average torque developed and of amplitude of torque oscillation.

\section{DISCUSSION}

Physiological sympathetic activation by CPT did not produce a potentiation of the contraction in any of the tested muscles. Conversely, a significant decrease in twitch amplitude was observed in tibialis anterior while only a trend towards twitch shortening was observed in the calf muscles. This set of results indicates that a weakening rather than a potentiating effect has been induced by sympathetic activation.
Potentiation vs. weakening. In vitro studies $(5,12)$ have elucidated that adrenergic agonists may modulate the contractile machinery of skeletal muscles in two ways: 1 ) by increasing the reuptake of $\mathrm{Ca}^{2+}$ in the sarcoplasmatic reticulum, thus shortening the twitch duration (positive lusitropism) and resulting in a weakening effect, this mechanisms being present in type I fibers only; and 2) by augmenting the release of $\mathrm{Ca}^{2+}$ from the SR, thus producing a twitch of bigger amplitude, which is a potentiation of the contraction, this mechanisms is present in both fiber types although it has been observed mostly in fast-twitch muscles $(1,3,4)$. These classic studies, performed on animal models, already pointed out that higher doses of Epi or $\beta_{2}$-agonist had to be administered to elicit a potentiating effect in fast-twitch muscles with respect to the
Fig. 5. Analysis of torque developed by stimulation with double pulses of the tibial nerve (experiment 2). A: superimposition of the torque produced by the 21 doublets at increasing ISI in 1 subject in a control condition. $B, C$, and $D$ : effect on amplitude, time to peak, and half relaxation time of the torque produced by the second pulse in the doublet is displayed vs. the ISI (ms) for 3 conditions. Each trace is the average of all individual traces $(n=9)$, and ctrl is the average of the 3 control conditions. Abbreviations are as in Fig. 3.
A
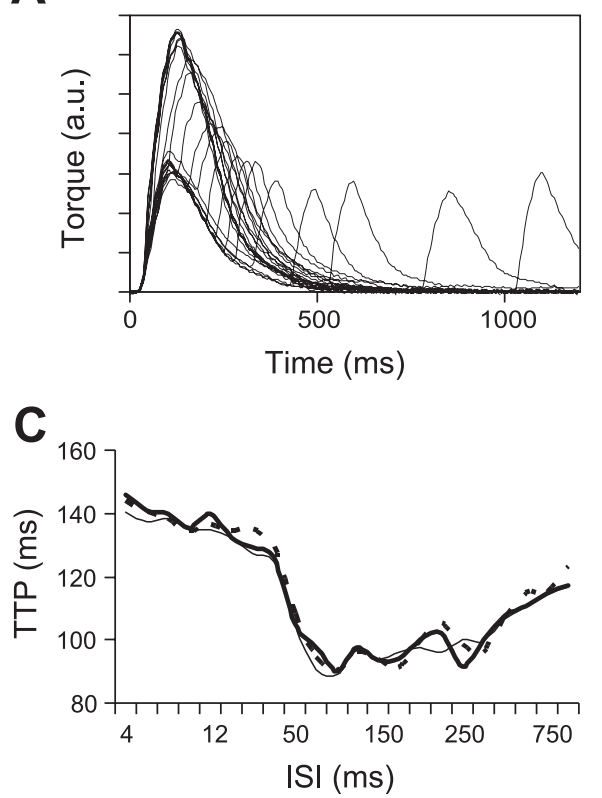

B

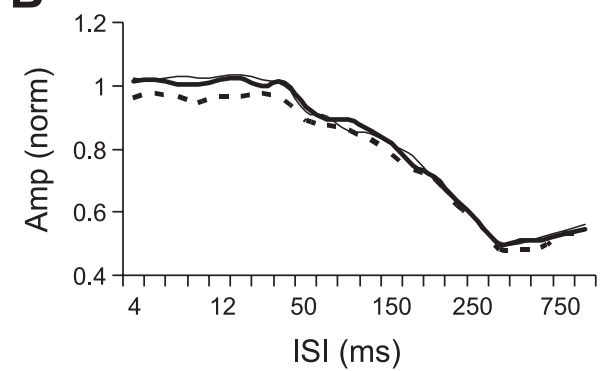

D

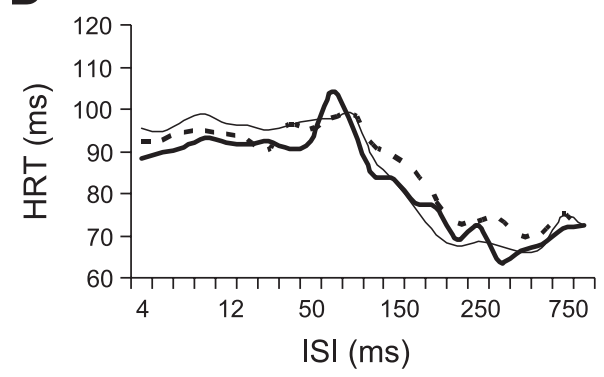


dose required to elicit the weakening effect in slow-twitch muscles $(1,3,4)$. This difference can be partly attributed to the fact that type I fibers have a higher density of adrenergic receptor than type II $(15,22)$. On the other hand, it is a widely held view that the sympathetic nervous system potentiates the contraction of skeletal muscles $(9,11,33,36)$. This idea fits well with the other actions that the sympathetic nervous system exerts, particularly on the cardiovascular system, to support intense muscle work and is appropriate in a context of fight or flight. However, it must be emphasized that no human study currently evidenced the occurrence of sympathetic-mediated potentiation of skeletal muscles. Moreover, many animal and in vitro studies that report catecholamine-mediated potentiation refer to muscles that were previously fatigued $(3,23)$ or to muscle fibers immersed in an iperkalemic medium (13). The force potentiation of fatigued muscles, also called "anti-fatigue" effect, is based on the recovery of cell excitability by Epi-induced potentiation of the $\mathrm{Na}^{+}-\mathrm{K}^{+}$pump $(3,13,29)$ and does not mediate the positive inotropic effect observed in resting fast-twitch muscles $(1,3,4)$.

In the present study, CPT failed to induce any potentiation in either tibialis anterior or calf muscles, although the same stressor was adequate to induce the weakening effect in low threshold, presumably type I, motor units of the tibialis anterior (28). This supports the concept that the positive inotropic effect has a higher threshold of activation than the weakening effect.

These results also support and integrate the only investigation in humans about the effects of exogenous (not spontaneously released, as in the present study) Epi on muscle contractility by Marsden and Meadows (21). Although their interest was mostly focused on the tremor-genic action of Epi, the authors evidenced a weakening adrenergic effect in both the calf muscles ( 5 subjects) and the adductor pollicis ( 3 subjects). In particular, they showed a reduction in HRT of the twitch force in the calf muscles $(\sim 15 \%)$, and no significant effect on the twitch force of adductor pollicis but a decrease in the force of subtetanic contraction (10-Hz stimulation), as we did observe for tibialis anterior.

The protocol adopted in the present study included electrical stimulation by paired stimuli at varying ISI within the range $4-1,000 \mathrm{~ms}$. This stimulation pattern was previously employed for the investigation of the velocity recovery function of muscle fibers (18) and of twitch summation (17). It was adopted in the present study for two reasons: 1 ) the response to the doublet at short ISI is stronger than the single twitch and thus provides an improved signal-to-noise ratio for the detection of changes in muscle contractility; and 2) possible increase/decrease in twitch duration, resulting in increased/decreased twitch fusion, would have been evidenced by rightward/leftward shift of the torque amplitude vs. ISI curve in this stimulation pattern.

The reduction in single twitch amplitude in tibialis anterior was confirmed by the reduction in the response to paired stimuli $(4<$ ISI $<30$; Fig. $3 B)$ as well as by a reduction in the torque developed by burst stimulation at the different frequencies. This supports the interpretation that sympathetic activation by CPT produced a weakening effect. In fact, in many animal studies a marked decrease of the twitch amplitude was observed in response to Epi injection, as a consequence of the lusitropic effect occurring in type I fibers $(1,3,4)$. It is possible that this latter effect was masked in the present study due to the coactivation of unresponsive or differently responding type II fibers in tibialis anterior. A decrease in HRT was instead observed in response to CPT-induced sympathetic activation in our previous study where single, low-threshold, presumably type I motor units were investigated (28), while an increased HRT was observed in the tibialis anterior of healthy subjects in response to blockade of $\beta$-adrenergic receptors (2).

In the soleus muscle, the reduction in HRT observed in the single twitch was also observed in response to close paired stimuli ( $4 \leq$ ISI $\geq 30$; Fig. $5 D$ ), although the effect was probably too weak to reach statistical significance and to produce appreciable changes in the amplitude vs. ISI curve as well as in the burst contractions.

Electrical stimulation does not allow selective recruitment of type II muscle fibers. Therefore, the possibility exists that a potentiating effect occurring in type II fibers was canceled by concomitant weakening effects in type I fibers when the muscle is composed of a balanced proportion of the two types of fibers. In fact, Bowman and Zaimis (4) observed clear cut potentiation in the fast-twitch tibialis muscle and marked weakening in the slow-twitch soleus muscle of the cat intravenously injected with Epi, while minor effects were observed in plantaris and gastrocnemius muscles characterized by a more balanced fiber type composition. In humans, both tibialis anterior and soleus muscles have a preponderance of type I fibers, so the possibility cannot be excluded that potentiation effects have been canceled by weakening effects occurring in these fibers. On the other hand, selective activation of type II fibers is also unlikely to occur in voluntary contractions since the orderly recruitment of motor units according to the size principle predicts that type I motor units are recruited first (14). On this basis, the possible ergogenic action of catecholamines would anyway hardly become functionally meaningful, given that most human skeletal muscles have a large percentage of type I fibers.

A possible complication in the interpretation of our results is that greater sympathetic activation may be required for observing potentiating effects on muscle fiber contractility than that provided by CPT. This opens for a potential functional role of potentiation of contractility at higher activation levels of the sympathetic system. However, CPT, which provokes a consistent increase in arterial blood pressure and in plasma catecholamines (31), is a stimulus that is already quite difficult to sustain: VAS pain scores up to 9.2 were reported in the present study, while in previous studies some subject could not tolerate the pain level and interrupted the test before completion (28). Nevertheless, the involvement of the sympathoadrenal axis in the stress response is stressor dependent $(27,29,31)$ and it cannot be excluded that the ergogenic effect can be better detected in response to other experimental stimuli.

CPT is also reported to increase muscle sympathetic activity (10) and to reduce blood flow to resting limb muscles (37). Reduction in the blood supply was shown to decrease the muscle force capacity in fatigued and resting muscles $(25,38)$. Although it is unlikely that a small reduction in blood flow [20\% in the study of Wray et al. (37)] for a duration of few minutes might have affected the force capacity of a resting muscle, this possibility cannot be completely excluded. On the other hand, the increase of vascular resistances and limitation of blood flow to different organs, including skeletal muscles, are part of sympathetic activation patterns, so this indirect weakening effect of sympathetic activation on muscle force 
capacity should also be taken into account when considering functional effects.

Limitations. In a context of generalized sympathetic activation, motor control may be affected at different central and peripheral levels. To focus the investigation on the sympathetic effects on muscle contractility, this study was based on electrically stimulated contractions that provide a standardized model to reproduce muscle contraction with high repeatability and independence from the central motor command. This choice was motivated by the observation that the motor command adapted and compensated for changes occurring at the effector level during sympathetic activation (28). However, limitations of this approach need to be taken into account.

Supramaximal percutaneous nerve stimulation, which is generally adopted to obtain full muscle activation, may be rather painful. The pain produced by the electric shocks is a powerful stimulus for sympathetic activation $(6,26)$, just like the cold-induced pain at the immersed hand during the CPT (26). Providing pain stimuli throughout all sessions would have raised basal sympathetic outflow, thus attenuating or masking the effect of CPT under investigation. For this reason, the intensity of stimulation was not increased beyond low/moderate pain levels. This, however, resulted in submaximal stimulation of the tibial nerve in most subjects, which may have introduced some additional variability in the data and decreased the sensitivity in the technique, especially in experiment 2.

In addition, nerve stimulation does not allow to selectively stimulate a single muscle. This is particularly true for stimulation of the tibial nerve, which leads to the contraction of other muscle groups in addition to the soleus, including the gastrocnemius muscle. The $100^{\circ}$ knee flexion position was indeed adopted to disengage the gastrocnemius muscle, thus limiting its contribution. However, some extra force contribution might have remained and be possibly responsible for the nonsmooth shape of the twitch in some subjects (21). These two limitations may partly account for the lack of effects observed in experiment 2.

Functional implications. The present results do not support the presence of a sympathetically mediated ergogenic effect on muscle contractility. Conversely, they confirm the presence of a weakening effect even in the relatively fast-twitch tibialis anterior muscle. How does a weakening effect comply with the needs of a fight-or-flight response and with the numerous studies reporting increased muscle performance after administration of $\beta_{2}$-adrenergic agonists?

First of all, the maximum force capacity of the muscle is not impaired by the lusitropic effect (3), although an increased driving frequency would be necessary to attain the same force (28). Secondly, the possibility to produce rapid alternating movements, as those required in fight and flight, should be improved by faster muscle relaxation (29). On this basis, it is not surprising that significant increases in performance after administration of $\beta_{2}$-agonists has been reported almost only for short-lasting and rapid tasks, such as the Wingate test $(8,20$, $32,34)$.

Besides the generalized sympathetic activation that characterizes the fight-or-flight response, sympathetic outflow is known to be highly differentiated to different organs and tissues depending on the context or stimulus according to the so-called autonomic "signature," which also concerns the bal- ance between sympathoneural and sympathoadrenal pathways $(24,27,30)$. Thus sympathetic modulation of muscle contractility should also be expected to occur in other situations activating the sympathoadrenal axis. In this respect and in support of the current view, it is interesting to mention the anecdotal reports of back and leg muscle weakness during states of fear and anxiety as well as in response to adrenaline infusion (3).

Conclusion. In conclusion, for the first time the occurrence of an adrenergic-mediated positive inotropic action has been sought during physiological sympathetic activation. The CPT failed to induce any ergogenic effect while producing instead some decrease in the electrically stimulated muscle force. Peripheral effects of either direct (on the muscle fibers) or indirect (secondary to circulatory changes) adrenergic actions are presumed to underlie the weakening sympathetic action.

\section{ACKNOWLEDGMENTS}

We are grateful to Mara Rolando, Fabrizio Picariello, Corrado Cescon, and Ernest N. Kamavuako for help with experimental procedures and data analysis.

\section{GRANTS}

This work was supported by grants from Regione Piemonte (Ricerca sanitaria finalizzata 2007-2009).

\section{DISCLOSURES}

No conflicts of interest, financial or otherwise, are declared by the author(s).

\section{REFERENCES}

1. Al-Jeboory AA, Marshall RJ. Correlation between the effects of salbutamol on contractions and cyclic AMP content of isolated fast-and slowcontracting muscles of the guinea pig. Naunyn Schmiedebergs Arch Pharmacol 305: 201-206, 1978.

2. Alway SE, Hughson RL, Green HJ, Patla AE. Human tibialis anterior contractile responses following fatiguing exercise with and without betaadrenoceptor blockade. Clin Physiol 8: 215-225, 1988.

3. Bowman WC. Effects of adrenergic activators and inhibitors on the skeletal muscles. In: Handbook of Eperimental Pharmacology, Adrenergic Activators and Inhibitor, edited by Szekeres L. Berlin: Springer, 1980, p. $47-128$.

4. Bowman WC, Zaimis E. The effects of adrenaline, noradrenaline and isoprenaline on skeletal muscle contractions in the cat. J Physiol 144: 92-107, 1958.

5. Cairns SP, Dulhunty AF. The effects of beta-adrenoceptor activation on contraction in isolated fast- and slow-twitch skeletal muscle fibres of the rat. Br J Pharmacol 110: 1133-1141, 1993.

6. Christou EA, Jakobi JM, Critchlow A, Fleshner M, Enoka RM. The 1to $2-\mathrm{Hz}$ oscillations in muscle force are exacerbated by stress, especially in older adults. J Appl Physiol 97: 225-235, 2004.

7. Collomp K, Candau R, Millet G, Mucci P, Borrani F, Prefaut C, De Ceaurriz J. Effects of salbutamol and caffeine ingestion on exercise metabolism and performance. Int J Sports Med 23: 549-554, 2002.

8. Collomp K, Le Panse B, Portier H, Lecoq AM, Jaffre C, Beaupied H, Richard O, Benhamou L, Courteix D, De Ceaurriz J. Effects of acute salbutamol intake during a Wingate test. Int J Sports Med 26: 513-517, 2005.

9. Dienstbier RA. Behavioral correlates of sympathoadrenal reactivity: the toughness model. Med Sci Sports Exerc 23: 846-852, 1991.

10. Fagius J, Karhuvaara S, Sundlof G. The cold pressor test: effects on sympathetic nerve activity in human muscle and skin nerve fascicles. Acta Physiol Scand 137: 325-334, 1989.

11. French DN, Kraemer WJ, Volek JS, Spiering BA, Judelson DA, Hoffman JR, Maresh CM. Anticipatory responses of catecholamines on muscle force production. J Appl Physiol 102: 94-102, 2007.

12. Ha TN, Posterino GS, Fryer MW. Effects of terbutaline on force and intracellular calcium in slow-twitch skeletal muscle fibres of the rat. $\mathrm{BrJ}$ Pharmacol 126: 1717-1724, 1999. 
13. Hansen AK, Clausen T, Nielsen OB. Effects of lactic acid and catecholamines on contractility in fast-twitch muscles exposed to hyperkalemia. Am J Physiol Cell Physiol 289: C104-C112, 2005.

14. Henneman E, Somjen G, Carpenter DO. Functional significance of cell size in spinal motoneurons. J Neurophysiol 28: 560-580, 1965.

15. Jensen J, Brennesvik EO, Bergersen H, Oseland H, Jebens E, Brors $O$. Quantitative determination of cell surface beta-adrenoceptors in different rat skeletal muscles. Pflügers Arch 444: 213-219, 2002.

16. Johnson MA, Polgar J, Weightman D, Appleton D. Data on the distribution of fibre types in thirty-six human muscles. An autopsy study. J Neurol Sci 18: 111-129, 1973.

17. Kamavuako EN, Farina D. Time-dependent effects of pre-conditioning activation on muscle fiber conduction velocity and twitch torque. Muscle Nerve 42: 547-555, 2010.

18. Kamavuako EN, Hennings K, Farina D. Velocity recovery function of the compound muscle action potential assessed with doublet and triplet stimulation. Muscle Nerve 36: 190-196, 2007.

19. Kindermann W. Do inhaled beta(2)-agonists have an ergogenic potential in non-asthmatic competitive athletes? Sports Med 37: 95-102, 2007.

20. Le Panse B, Arlettaz A, Portier H, Lecoq AM, De Ceaurriz J, Collomp K. Effects of acute salbutamol intake during supramaximal exercise in women. Br J Sports Med 41: 430-434, 2007.

21. Marsden CD, Meadows JC. The effect of adrenaline on the contraction of human muscle. J Physiol 207: 429-448, 1970.

22. Martin WH III, Coggan AR, Spina RJ, Saffitz JE. Effects of fiber type and training on $\beta$-adrenoceptor density in human skeletal muscle. Am J Physiol Endocrinol Metab 257: E736-E742, 1989.

23. Mikkelsen UR, Gissel H, Fredsted A, Clausen T. Excitation-induced cell damage and $\beta_{2}$-adrenoceptor agonist stimulated force recovery in rat skeletal muscle. Am J Physiol Regul Integr Comp Physiol 290: R265R272, 2006.

24. Morrison SF. Differential control of sympathetic outflow. Am J Physiol Regul Integr Comp Physiol 281: R683-R698, 2001.

25. Murthy G, Hargens AR, Lehman S, Rempel DM. Ischemia causes muscle fatigue. J Orthop Res 19: 436-440, 2001.

26. Nordin M, Fagius J. Effect of noxious stimulation on sympathetic vasoconstrictor outflow to human muscles. J Physiol 489: 885-894, 1995.
27. Pacak K, Palkovits M. Stressor specificity of central neuroendocrine responses: implications for stress-related disorders. Endocr Rev 22: 502 548, 2001.

28. Roatta S, Arendt-Nielsen L, Farina D. Sympathetic-induced changes in discharge rate and spike-triggered average twitch torque of low-threshold motor units in humans. J Physiol 586: 5561-5574, 2008.

29. Roatta S, Farina D. Sympathetic actions on the skeletal muscle. Exerc Sport Sci Rev 38: 31-35, 2010.

30. Roatta S, Mohammed M, Passatore M. Detecting activation of the sympatho-adrenal axis from haemodynamic recordings, in conscious rabbits exposed to acute stress. Acta Physiol (Oxf) 201: 323-337, 2011.

31. Robertson D, Johnson GA, Robertson RM, Nies AS, Shand DG, Oates JA. Comparative assessment of stimuli that release neuronal and adrenomedullary catecholamines in man. Circulation 59: 637-643, 1979.

32. Signorile JF, Kaplan TA, Applegate B, Perry AC. Effects of acute inhalation of the bronchodilator, albuterol, on power output. Med Sci Sports Exerc 24: 638-642, 1992.

33. Tod D, Iredale F, Gill N. "Psyching-up" and muscular force production Sports Med 33: 47-58, 2003.

34. van Baak MA, Mayer LH, Kempinski RE, Hartgens F. Effect of salbutamol on muscle strength and endurance performance in nonasthmatic men. Med Sci Sports Exerc 32: 1300-1306, 2000.

35. Van Der Heijden HF, Zhan WZ, Prakash YS, Dekhuijzen PN, Sieck GC. Salbutamol enhances isotonic contractile properties of rat diaphragm muscle. J Appl Physiol 85: 525-529, 1998

36. Williams JH, Barnes WS. The positive inotropic effect of epinephrine on skeletal muscle: a brief review. Muscle Nerve 12: 968-975, 1989.

37. Wray DW, Donato AJ, Nishiyama SK, Richardson RS. Acute sympathetic vasoconstriction at rest and during dynamic exercise in cyclists and sedentary humans. J Appl Physiol 102: 704-712, 2007.

38. Wright JR, McCloskey DI, Fitzpatrick RC. Effects of systemic arterial blood pressure on the contractile force of a human hand muscle. J Appl Physiol 88: 1390-1396, 2000.

39. Zouhal H, Jacob C, Delamarche P, Gratas-Delamarche A. Catecholamines and the effects of exercise, training and gender. Sports Med 38: 401-423, 2008. 